Article

\title{
A Modified Bird-Mating Optimization with Hill-Climbing for Connection Decisions of Transformers
}

\author{
Ting-Chia Ou ${ }^{1, *}$, Wei-Fu Su ${ }^{2}$, Xian-Zong Liu ${ }^{3}$, Shyh-Jier Huang ${ }^{3}$ and Te-Yu Tai ${ }^{3}$ \\ 1 Institute of Nuclear Energy Research, Taoyuan 32546, Taiwan \\ 2 Department of Electrical Engineering, Kun Shan University, Tainan 71070, Taiwan; wfsu@mail.ksu.edu.tw \\ 3 Department of Electrical Engineering, National Cheng Kung University, Tainan 70101, Taiwan; \\ zong@ciap5.ee.ncku.edu.tw (X.-Z.L.); clhuang@mail.ncku.edu.tw (S.-J.H.); allextai1992@gmail.com (T.-Y.T.) \\ * Correspondence: tcou@iner.gov.tw; Tel.: +886-2-8231-7717 (ext. 6389)
}

Academic Editor: Issouf Fofana

Received: 23 May 2016; Accepted: 18 August 2016; Published: 23 August 2016

\begin{abstract}
This paper endeavors to apply a hybrid bird-mating optimization approach to connection decisions of distribution transformers. It is expected that with the aid of hybrid bird-mating approach, the voltage imbalance and deviation can be mitigated, hence ensuring a satisfactory supplying power more effectively. To evaluate the effectiveness of this method, it has been tested through practical distribution systems with comparisons to other methods. Test results help confirm the feasibility of the approach, serving as beneficial references for the improvement of electric power grid operations.
\end{abstract}

Keywords: bird-mating optimization; voltage imbalance; and voltage deviation

\section{Introduction}

Three-phase and single-phase loads are sometimes supplied by transformers in the distribution system [1-3]. However, different connections of transformers may cause severe phase voltage imbalance, resulting in a larger ground current and bringing extra power losses. With consideration of transformer costs and loss reduction, the connection of transformers and negative sequence (N-Seq) current injection into utility generators have been deemed a feasible alternative to improve such system imbalance [4,5]. A systematic study with an effective solution approach is crucial to maintain a good quality of supplying power, thereby motivating the study made in this paper.

Several improved schemes such as an energy storage system [6], static compensator (STATCOM) control [7,8], feeder cross-section increment, capacitor installation [9], flexible AC transmission system (FACTS) devices [10], and network reconfiguration [11] were proposed to deal with this three-phase imbalance problem [12-14]. Compared to the additional investment for equipment purchasing, network reconfiguration became an economically feasible alternative, which can be performed by solely changing the connection of distribution transformers. The phase-switching work can be practically incorporated below: (a) Choose the transformer to be ready for the change of phase connection; (b) Perform the fuse link to disconnect the regional power supply; (c) Ensure the attachment of the grounding copper rod; (d) Execute the phase-switching work following the confirmation of the above steps; and (e) Restore the power supply with the operation of fuse link, and complete the phase-changing work. It is also worth noting that the limitations in changing the connection should be taken into consideration since the load information varies with time, yet the phase-changing work is not real-time because it needs field labors. Previously, by formulating the phase-connection problem as a linear objective function, classical methods of using mixed-integer programming were employed to solve such imbalance problems $[15,16]$. The results were proved effective, yet it is arguable since the problem was handled as a linear function with approximation. 
In view of aforementioned issues, several heuristic methods were previously suggested [17-19]. An intelligent approach of hybrid bird-mating optimization is also proposed in this study to solve the transformer connection problem. By mimicking the courtship behavior observed in the mating process of birds, different strategies of conventional bird-mating optimization was developed [20]. Feasibility of such a method was validated yet failed to locate the global minima for complex multi-minima functions because of limited capability of exploiting more areas. To improve such a demerit, this paper further embeds the bird-mating optimization with a local random search procedure and diversity measurement, by which the connection work in a distribution grid is anticipated with a higher confidence. Moreover, with the systematic computation of the proposed method, it can be included with commercial software for industry applications, creating a potential for extending to distribution automations.

\section{Problem Description}

In this paper, the objectives of connection adjustment of transformers are to improve the voltage imbalance of each node and to minimize the voltage deviation while satisfying equality and inequality constraints of network operation. All functions and constraints are expressed as a multi-objective optimization problem, which are detailed below.

Connections of distribution transformers in a distribution network of Taiwan can be largely divided into 'single-phase three-wired' and 'three-phase four-wired'. When the single-phase three-wired connection is made, transformers with mid-tap grounded are often adopted for lighting loads. With a three-phase four-wired connection, a considerable number of transformers are utilized to supply three-phase and single-phase loads for economic benefits and space-saving.

The objective functions of this study are formulated so as to minimize the total voltage imbalance ratio and deviation ratio. These functions and constraints are investigated as follows. Firstly, the minimization of voltage imbalance ratio helps improve the voltage profile. A lower value of voltage imbalance ratio implies a system with a better balance. By summing up voltage imbalance ratios of all nodes, an objective function can be expressed below

$$
\begin{aligned}
& \text { Minimize } f_{1}(\boldsymbol{x}, \boldsymbol{u})=\sum_{i=1}^{N} V U R_{i} \\
& =\sum_{i=1}^{N} \frac{\max \left(|| V_{i}^{x y}\left|-V_{i}^{\text {avg }}\right|,|| V_{i}^{y z z}\left|-V_{\text {avg }}\right|,|| V_{i}^{z x}\left|-V_{i}^{\text {avg }}\right|\right)}{V_{i}^{\text {avg }}} \times 100 \%
\end{aligned}
$$

where $V U R_{i}$ is the voltage imbalance ratio of $i$-th bus, $V_{i}^{x y}, V_{i}^{y z}$, and $V_{i}^{z x}$ are three-phase line voltages of $i$-th bus, $V_{i}^{a v g}$ is the average line voltage among $V_{i}^{x y}, V_{i}^{y z}$, and $V_{i}^{z x}$, and $N$ is the number of nodes in a distribution system [21,22]. The vector of dependent variables $x$ is expressed as

$$
x^{T}=\left[V_{1}, \ldots, V_{i}, \ldots, V_{N}, I_{1}, \ldots, I_{j}, \ldots I_{N_{l}}\right]
$$

where $V_{i}$ is the average phase voltage of the $i$-th node, and $I_{j}$ is the current on the $j$-th branch. The subscript $N$ defines the number of nodes, and $N_{l}$ represents the number of branches. The vector of control variables $u$ is written as

$$
u^{T}=\left[\chi_{1}^{O Y O D}, \ldots, \chi_{i}^{O Y O D}, \ldots, \chi_{N_{k}}^{O Y O D}, \chi_{1}^{\text {single }}, \ldots, \chi_{j}^{\text {single }}, \ldots \chi_{N_{s}}^{\text {single }}\right]
$$

where $\chi_{i}^{O Y O D}$ and $\chi_{j}^{\text {single }}$ are connections of the $i$-th transformer bank for each phase and the $j$-th single-phase transformer, $N_{k}$ is the number of transformer banks, and $N_{s}$ is the number of single-phase transformers.

Secondly, considering that the voltage profile may drift up and exceed the upper limit, the connection reconfiguration of a system with a better balance is suggested to help reduce the line 
current and lower the voltage deviation, hence preventing jeopardization of the system. Based on this minimization of voltage deviation, an objective function is formulated as follows

$$
\text { Minimize } f_{2}(\boldsymbol{x}, \boldsymbol{u})=\sum_{i=1}^{N} V D R_{i}
$$

where $V D R_{i}$ is the voltage deviation of the $i$-th node. By considering both voltage imbalance and voltage deviation, the paper is devoted to perform the connection of transformers while satisfying the operating constraints. Equality constraints consist of three-phase imbalanced power flow equations of a distribution system. For each state variable, the inequality constraints are expressed as follows.

Imbalanced three-phase voltages not only result in extra line losses, but also affect the lifetime of electrical equipment. The voltage imbalance ratio of each node needs to be restricted as $V U R_{i} \leq V U R_{\max }$, where $V U R_{\max }$ is the limit of voltage imbalance ratio, which is set at $2 \%$ in this study for a rigorous concern [23]. Next, a larger voltage deviation not only causes the load fluctuation, but also affects facility equipment. The voltage deviation needs to be regulated within a permissible range, $V D R_{i} \leq V D R_{\max }$, where $V D R_{\max }$ is the limit of voltage deviation ratio, which is set at $2.5 \%$ [24]. Since the over-voltage may jeopardize the customer, the voltage of each load bus should be operated within the interval of 1.05 and 0.95 , respectively.

\section{Proposed Approach}

This section introduces the hybrid bird-mating optimization with local random search and diversity measurement. The bird-mating optimization and its hybrid one are both detailed below.

\subsection{Bird-Mating Optimization}

The framework of bird-mating optimization is composed of bird species and their mating procedures. In this method, each bird represents a feasible solution for the problem, and genes of each bird are regarded as decision variables. The mating behaviors considered for female birds are parthenogenetic and polyandrous ones, and the promising genes gained through the process are solutions containing better objectives. This study considers the mating behaviors including monogamous, polygynous, and promiscuous processes. The corresponding computation mimicking each kind of bird-mating is discussed.

Monogamy is a form of relationship in which a male bird tends to copulate with a female bird. Polygyny is a form of plural marriage in which a male bird has a tendency to pair two or more female birds. As for the promiscuity, it refers to an unstable relationship between a male bird and his preferred female partner in the society. In this computation method, promiscuous birds are produced using stochastic process. Subsequently, concerning the polyandry, it is a form of relationship where a female bird tends to copulate with two or more male birds, and the offspring is simulated to be the same as polygynous birds generate in the method. Figure 1 shows the mating behaviors of all species of birds representing the location of distribution transformer, in which each bird string represents a set of primary phase-connections of distribution transformers. To confirm the search space, a bird string is encoded as $[0,1,2]$, representing the phase-connection of $[a, b, c]$ for single-phase transformers, and $[a b, b c, c a]$ for three-phase transformer banks, respectively. 


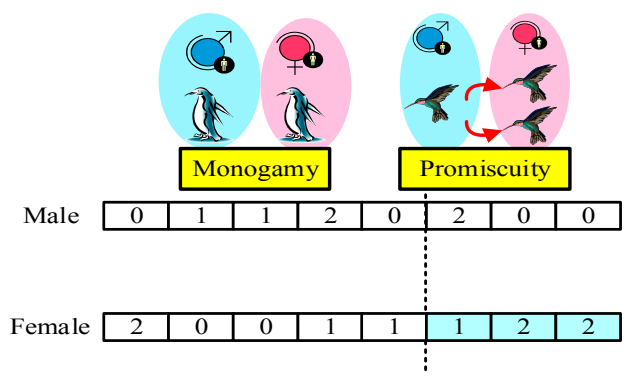

I

Brood

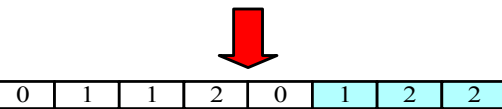

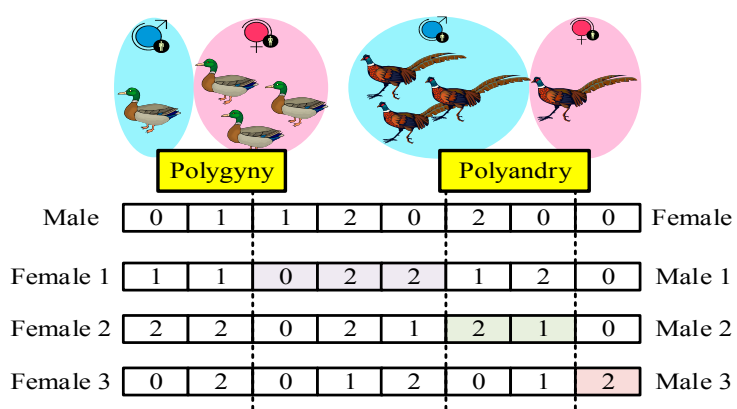

Brood
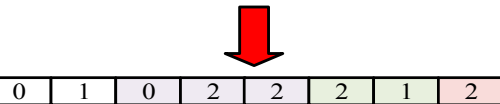

Figure 1. Mating process of all species of birds.

Different from the above mating, the parthenogenesis refers to an asexual reproduction in which a female bird raises brood without mating with any one male. Considering all of monogamous, polygynous, promiscuous, and polyandrous birds, Figure 2 conclusively shows their operation, meaning that the state of a bit is from 0 to 1 or 2 , or vice versa. In the proposed method, each parthenogenetic bird represents the better solution, attempting to pass her better genes to her brood with a smaller probabilistic disturbance. For the phase adjustment application in this paper, the lower bound of 0 stands for the "ab" phase-connection, the value of 1 is for the " $b c$ " phase-connection, and the upper bound of 2 is for the "ca" phase-connection.
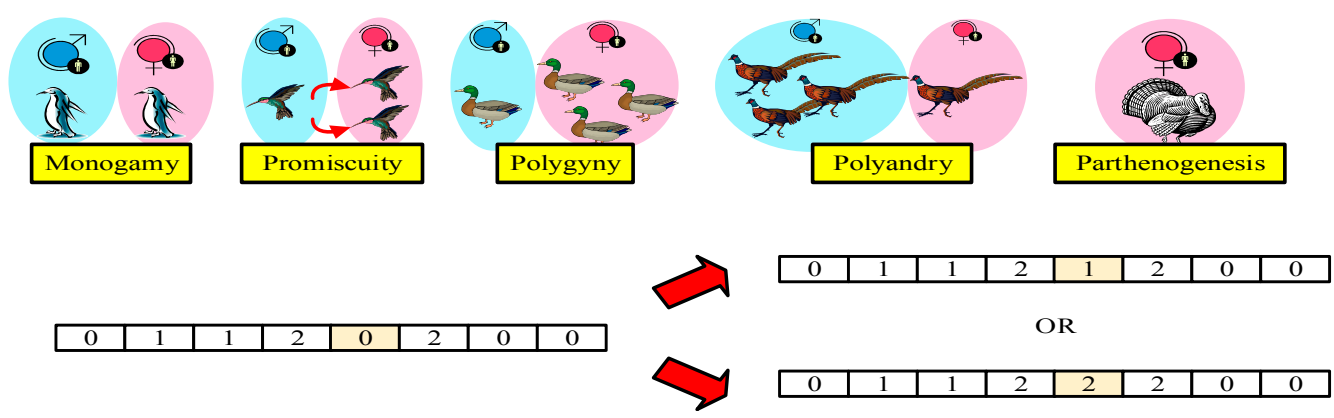

Figure 2. Mutation process of all species of birds.

\subsection{Hybrid Bird-Mating Optimization Approach}

The bird-mating optimization offers advantages of a simple concept, fewer parameters, and easy implementation. This approach is a good way of locating some promising areas with a better solution, yet it may fail to locate global minima because of limited capability of exploiting more areas. Mechanisms of diversity increment and local search are hence suggested to add, anticipating enhancing the solution performance of phase-connection of distribution transformers. A simple way of calculating the standard deviation of objective function values is adopted here in order to increase the diversity of the bird group. This standard deviation quantifies the amount of variation or dispersion of a set of data. When such a value decreases, it signals the promiscuous birds to generate more offspring to increase the diversity of the solution set, thus ensuring a sufficient diversity in the search space.

In the original approach, parthenogenetic birds are regarded as solutions with a higher possibility of evolving to be better ones. Yet, considering that the exploitation around these solutions may not be thoroughly performed, a local random search is added to the original approach [25]. Such a local search has been adopted in several methods with different formulations. The new work of this study is to combine the hill climbing with the bird-mating optimization. Namely, if the current best solution has no improvement through local exploitations, then a hill climbing method is activated to assist in 
the solution search. By combining these modifications, a hybrid bird-mating optimization approach is proposed. Computation procedures are detailed below.

\section{Computation of the Method}

Figure 3 shows the flowchart of the proposed hybrid bird-mating optimization approach. Main blocks of the method are discussed as follows. The computation starts with the data retrieval of distribution transformers, customer information, and line impedance. The parameters of bird society size, the initial mating probability, and the maximum number of iterations are all initialized.

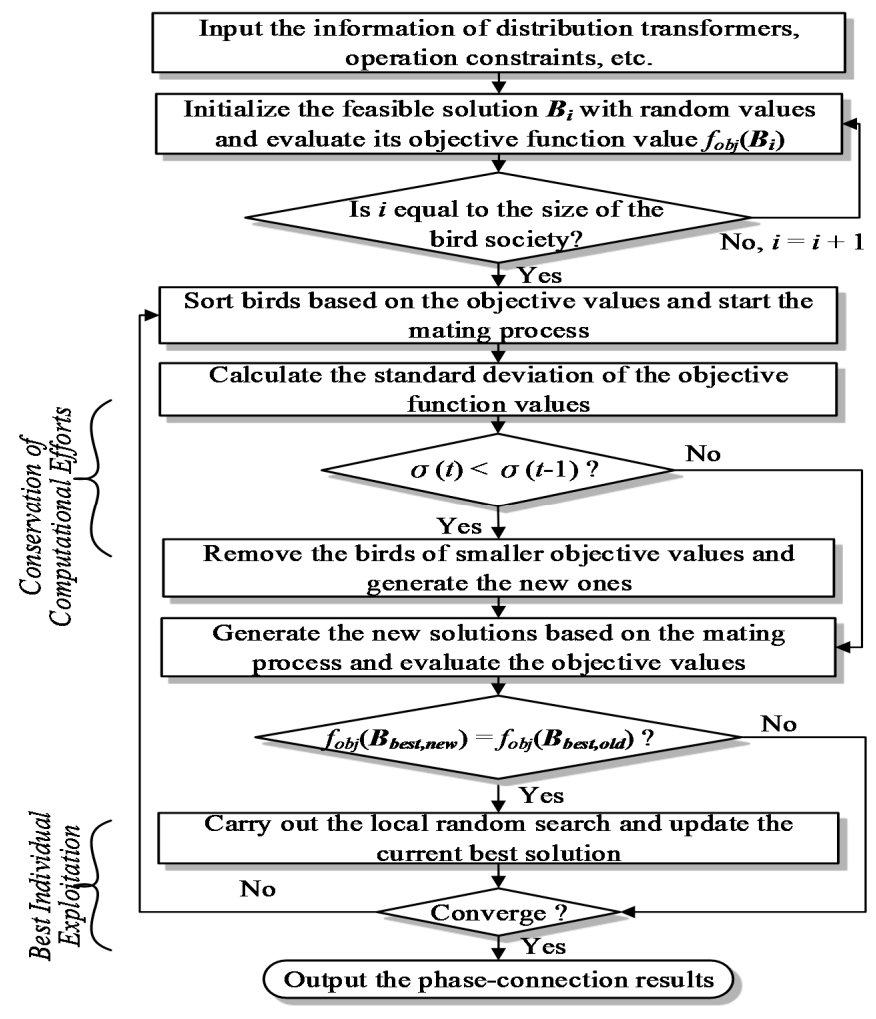

Figure 3. Flowchart of hybrid bird-mating optimization approach.

A society of $N$ birds is formed and each bird is composed of $M$ genes. A matrix of $\boldsymbol{B}=\left[\begin{array}{ll}\boldsymbol{B}_{1} & \boldsymbol{B}_{2} \\ \boldsymbol{B}_{3}\end{array}\right.$

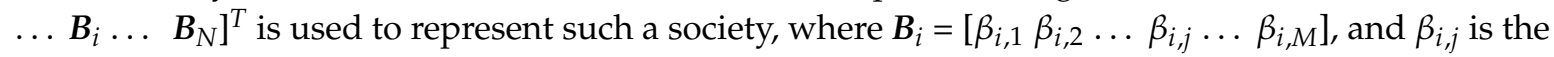
$j$-th gene of the $i$-th bird. In the society, each bird represents a potential solution to the problem, and each gene of a given society in matrix $\boldsymbol{B}$ is randomly assigned. Bird species are prescreened based on the objective value of $f_{o b j}\left(\boldsymbol{B}_{i}\right)$ to eliminate those with lower potentials. The standard deviation of all objective function is calculated. If the current standard deviation $\sigma(t)$ is smaller than the previous one $\sigma(t-1)$, then the promiscuous birds are randomly generated to increase the diversity of the solution set. These new set of solutions are next generated in the mating process. Next, the local random search is added such that the promising area can be better exploited. During the exploitation, if the objective value of the current best solution $f_{o b j}\left(\boldsymbol{B}_{\text {best }, \text { new }}\right)$ is equal to the previous $f_{o b j}\left(\boldsymbol{B}_{\text {best,old }}\right)$, it implies that the current best solution has no improvement. The local random search is then carried out in anticipation of finding a better one. This optimization process is terminated after concluding with no better solution occurred within a predefined number of iterations.

\section{Numerical Studies}

In order to confirm the effectiveness of the proposed bird-mating optimization for connection decision of transformers, the operation data acquired from real distribution systems of Taiwan Power 
Company (TPC) and the Institute of Nuclear Energy Research (INER) are served for validation. For all of tests, the results of using the proposed method are compared with those using genetic algorithm (GA), particle swarm optimization (PSO), and bird-mating optimization (BMO) algorithm. In the evaluation, the number of birds in the society is set at 100, in which the number of monogamous, polygynous, promiscuous, polyandrous, and parthenogenetic birds amount to 50, 30, 10, 5, and 5, respectively. The mating rate is set at 0.8 , the mutation rate is 0.1 , and the maximum number of allowed iterations is 1000. For both genetic algorithm and particle swarm optimization, the population size is 100 , the crossover probability is 0.8 , the mutation rate is 0.1 , and the cognitive and social parameters equal to 2 . These parameter settings are considered suitable for the problem concerned [26,27].

Figures 4 and 5 show the load patterns of feeders measured in an area of TPC. Because of capacitive effect caused by underground cables, the reactive power is fed to the secondary substation during some intervals, possibly bringing a higher voltage imbalance. Assumptions and descriptions of following scenarios are listed below. Case 1 considers the voltage imbalance ratio, case 2 considers the voltage deviation, and case 3 considers both of them. Each scenario along with its test results are examined as follows.

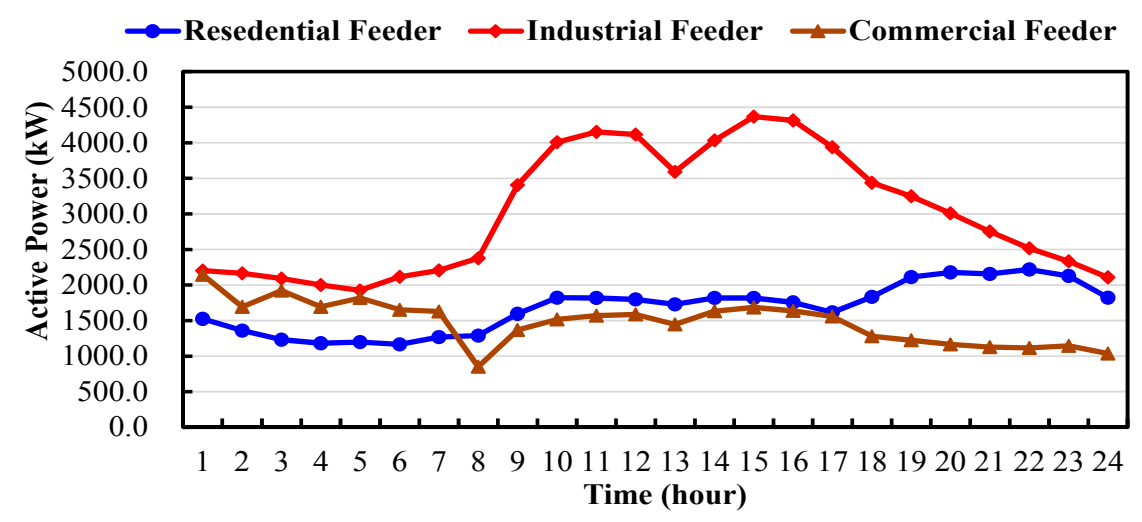

Figure 4. Active power curves of three test feeders.

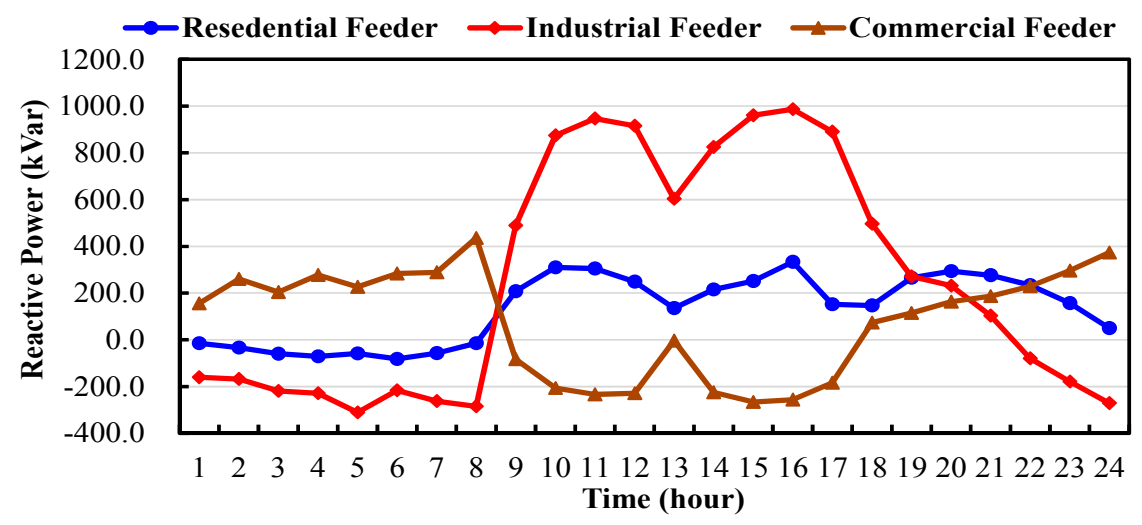

Figure 5. Reactive power curves of three test feeders.

\subsection{Case 1}

In this test, the proposed method is applied to an industrial feeder in the TPC test system. This feeder consists of seventeen $25 \mathrm{kVA}$, thirty-eight $50 \mathrm{kVA}$, forty-six $100 \mathrm{kVA}$, and nine $167 \mathrm{kVA}$ distribution transformers. The total feeder load is 4.36 MW and 2.22 MVar. There are 11 high-voltage customers connected to three-phase transformers.

Figure 6 shows the voltage imbalance ratio at each node before and after the phase adjustment considering minimizing the summation of voltage imbalance ratio at all nodes. It indicates that the voltage imbalance ratio can be decreased through the reconfiguration work. Figure 7 shows the 
convergence characteristics of different methods. Both bird-mating optimization (BMO) and hybrid bird-mating optimization (HBMO) have similar convergence features, which are better than other approaches. Because of added diversity and local search, the HBMO method is further improved. Figure 8 shows the daily voltage imbalance of initial connection and reconfiguration, where the largest imbalance often happens near the peak load in a day. Since the reconfiguration work is usually made around the peak load, the significant improvement occurs at hours 9-18 as the plot reveals. Table 1 shows that the proposed method can obtain the lowest Max.VUR\%. Comparing the Max.VUR\% of the regulation algorithms obtained by the proposed method with those prior to improvement, it can be seen that the Max.VUR\% fall by up to $34.5 \%$.

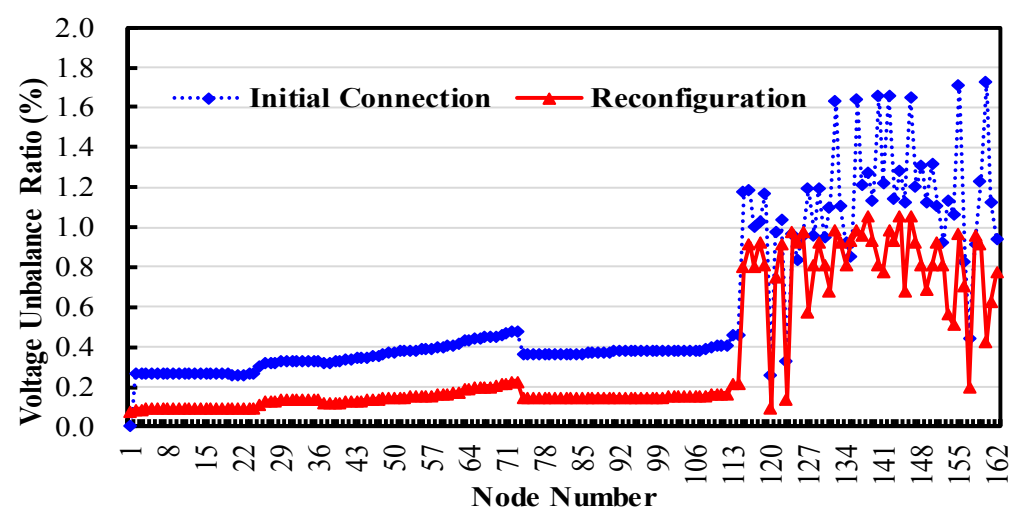

Figure 6. Comparison of the improvement in the voltage imbalance ratio for each node of the Taiwan Power Company (TPC) 162-node system.

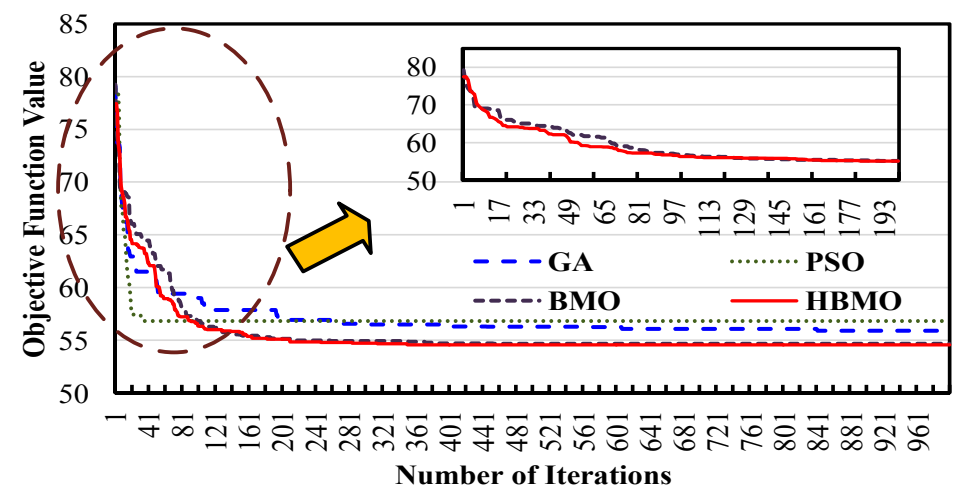

Figure 7. Comparison of convergence characteristics among methods.

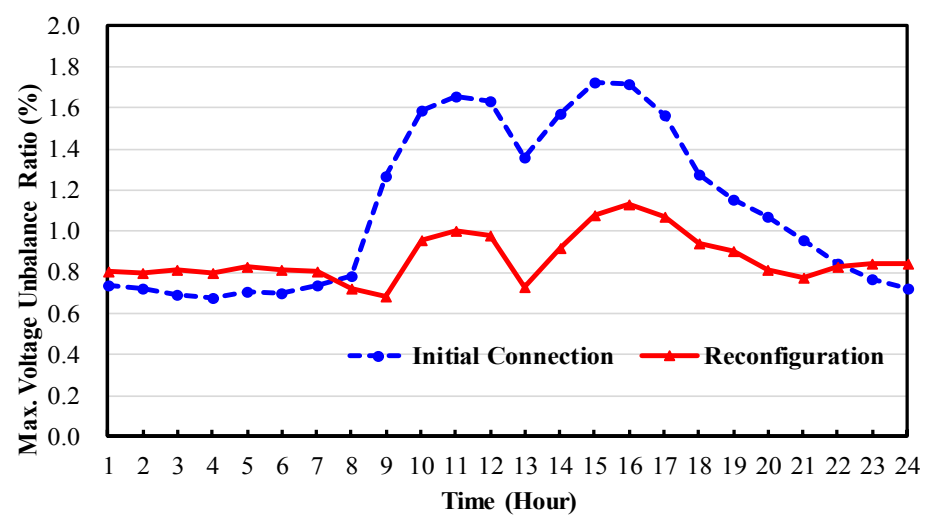

Figure 8. Comparison of the improvement in the Max. voltage imbalance ratio for the industrial feeder on a daily scale. 
Table 1. Comparison of algorithms considering the Max. voltage imbalance ratio as the single objective.

\begin{tabular}{ccccc}
\hline Max. VUR $\%$ & GA & PSO & BMO & HBMO \\
\hline Origin & $1.74 \%$ & $1.74 \%$ & $1.74 \%$ & $1.74 \%$ \\
Result & $1.16 \%$ & $1.16 \%$ & $1.14 \%$ & $1.14 \%$ \\
\hline
\end{tabular}

\subsection{Case 2}

In this case, the proposed method is applied to commercial feeders in a TPC test system. The feeders consists of nineteen $25 \mathrm{kVA}$, thirty-eight $50 \mathrm{kVA}$, fifty-three $100 \mathrm{kVA}$, and four $167 \mathrm{kVA}$ distribution transformers. Figure 9 shows the maximum voltage deviation at each node with and without the reconfiguration. The voltage deviation may occur due to the inclusion of renewable energy. The equivalent loads become more evenly distributed among phases after the reconfiguration. The line currents are reduced along with the mitigation of voltage rise. Figure 10 shows the daily voltage deviation gained from field measurements. After the reconfiguration, these increased voltage deviations can be effectively restricted.

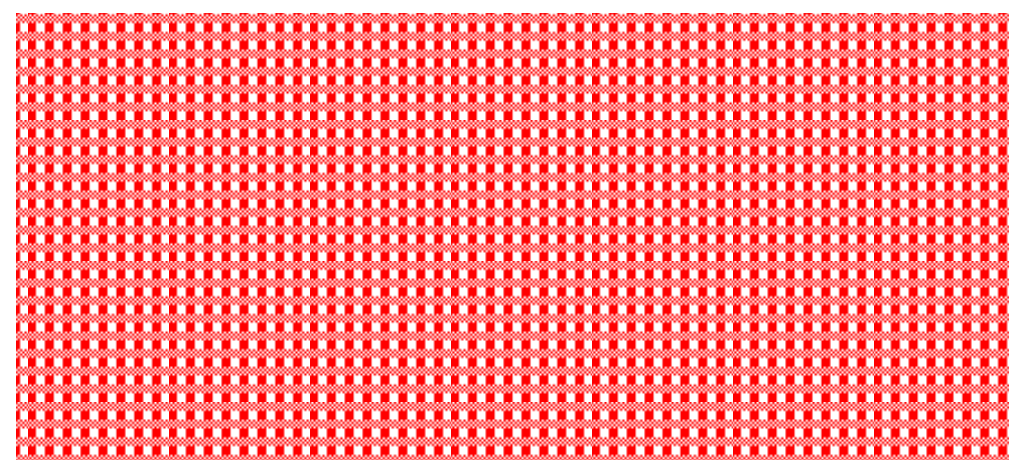

Figure 9. Comparison of voltage deviation for each node of the TPC test system.

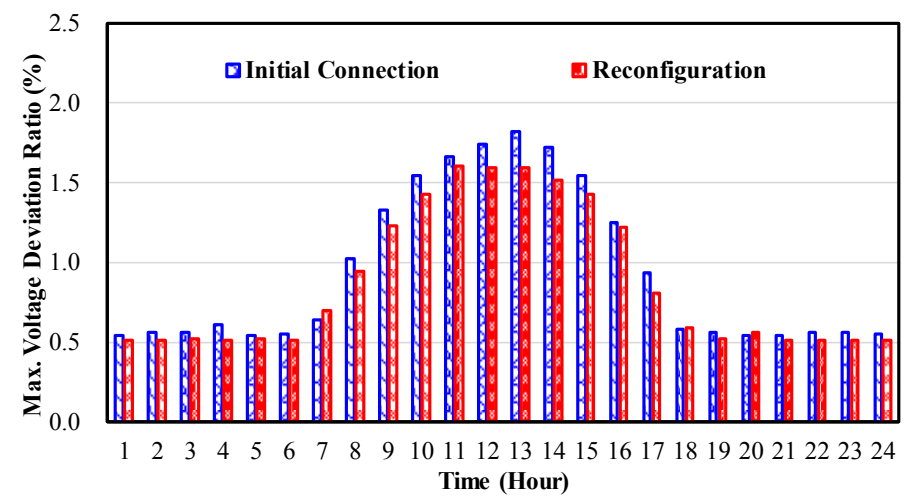

Figure 10. Daily voltage deviation ratio of initial connection and reconfiguration in the test feeder.

\subsection{Case 3}

In this case, the composite of maximum voltage imbalance ratio and voltage deviation ratio is concerned. The voltage imbalance of the system may become worse because of the connected renewable resources. Now for the case where the maximum voltage deviation ratio of $2.75 \%$, it can be controlled to be $2.5 \%$ after the reconfiguration. However, it is also suggested that if the voltage imbalance continues increasing, then the equipment such as energy storage system installation or additional transformer installation may be further required. It is worth mentioning that the suitable connection of transformers can be served as an alternative for utility engineers when the supplying quality is concerned. 
Figure 11 shows the daily voltage profile. In view of this voltage profile, the degree of imbalance is significant during the night because the peak load of this feeder occurs at 22:00. Through the reconfiguration, both daily voltage imbalance and deviation curve can be subsequently ameliorated at each hour, hence supporting the feasibility of the proposed method.

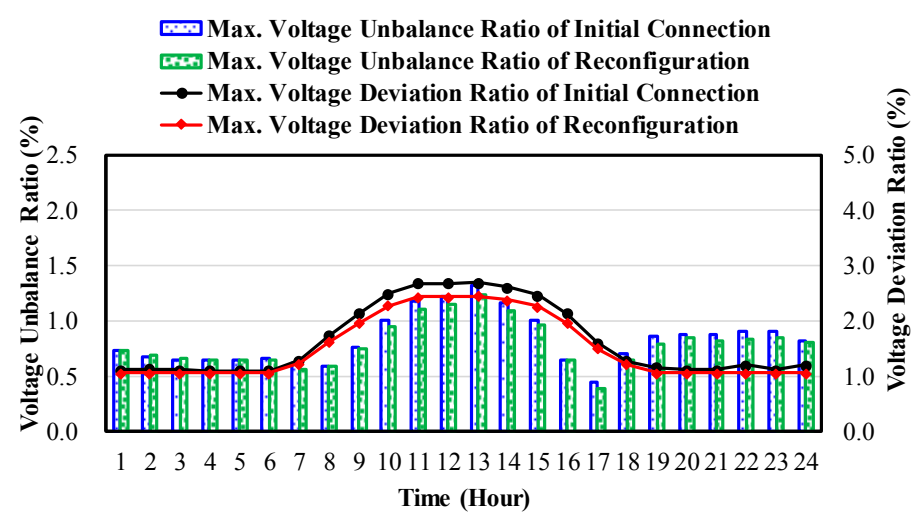

Figure 11. Daily voltage profile.

\subsection{Case 4}

A distribution network with renewable generation systems [28-30] in the Institute of Nuclear Energy Research (INER) was taken into account to perform the phase adjustment of distribution transformers in this case. Figure 12 shows the one-line diagram of the real $11.4 \mathrm{kV}$ distribution network in the INER. This system consists of 30 buses along with several hybrid energy resources, in which includes battery energy storage system (BESS), micro turbine generator (MTG), diesel generator (DG), photovoltaic system (PV), high concentration photovoltaic system (HCPV), vanadium redox battery (VRB), sodium sulfur fuel cell (SOFC), and wind turbine (WT), which are connected at Bus 18 in Figure 12. It is worth noting that all loads are supplied through open wye-open delta transformers in this simulation system.

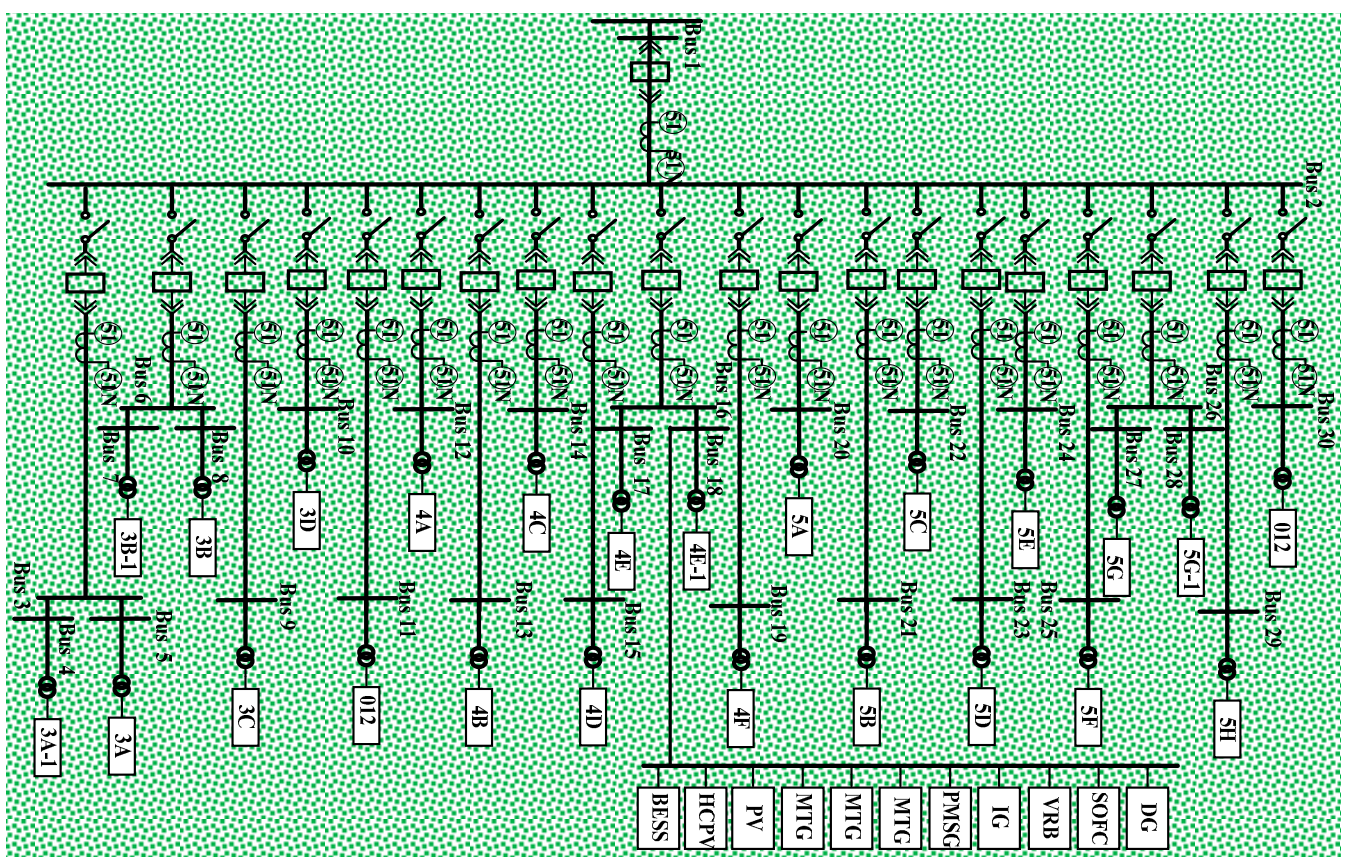

Figure 12. Single line diagram of $11.4 \mathrm{kV}$ substation of the Institute of Nuclear Energy Research (INER) 30-Bus system. 
Figure 13 shows the voltage imbalance ratio at each bus with and without the reconfiguration. The test shows that the voltage imbalance ratio of each bus not only decreases significantly after the rearranging phase of distribution transformers, but also satisfies the limit of allowable voltage balance ratio. Meanwhile, the total loss is also lowered down from $265.70 \mathrm{~kW}$ to $247.31 \mathrm{~kW}$ after the employment of the suggested approach. It is also worth noting that the aforementioned phase adjustment of distribution transformer is implemented as a human machine interface (HMI) to assist utility engineers in monitoring system operation. Figure 14 shows the display of HMI made by this paper. The functions of HMI include the single line diagram of $11.4 \mathrm{kV}$ substation of the INER 30-Bus system as well as the results with and without reconfiguration. The information of HMI including the voltage imbalance ratio of each bus, neutral line current, and total power loss are also displayed respectively. It is suitable for the utility engineer to supervise the grid with a higher convenience and efficiency.

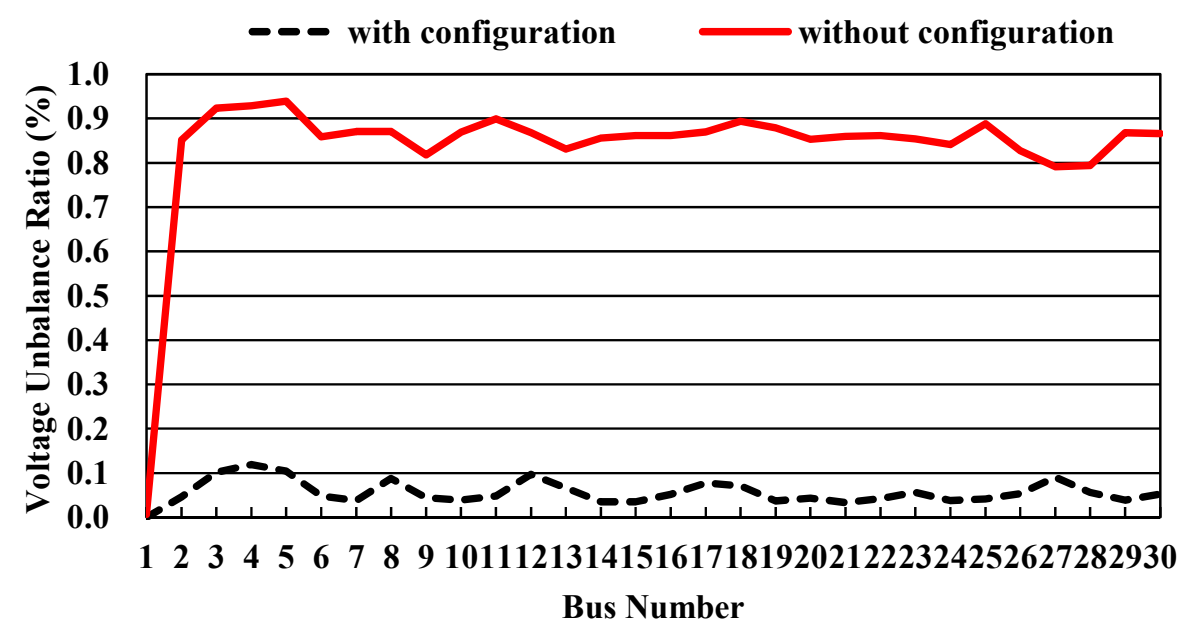

Figure 13. Comparison of the improvement in the voltage imbalance ratio for the INER 30-Bus system.

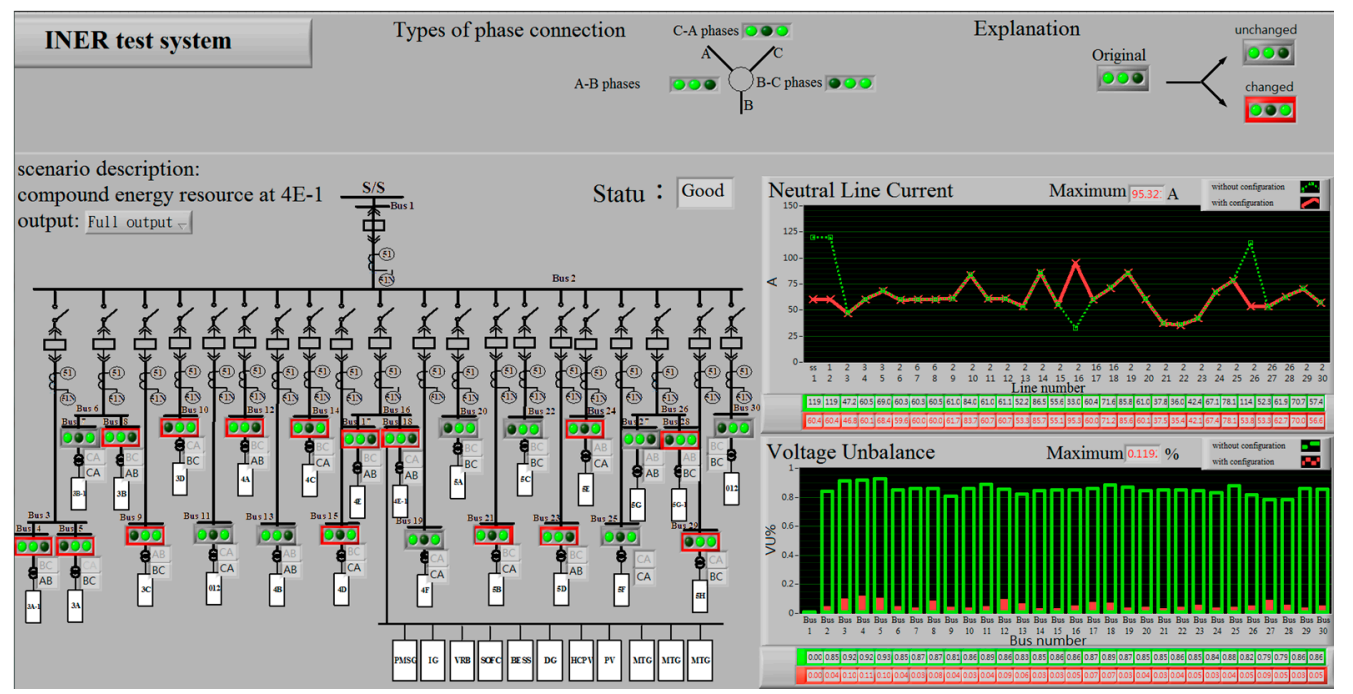

Figure 14. Comparison of the results of each Bus for the INER 30-Bus system considering reduction of voltage imbalance ratio and neutral line current with reconfiguration.

\section{Conclusions}

An approach using hybrid bird-mating optimization is proposed to enhance the phase-connection adjustment of distribution transformers in a grid. Through tests performed under three practical 
systems, this proposed approach has exhibited its capability of improving voltage imbalance and deviation. This work is being done in cooperation with the Institute of Nuclear Energy Research (INER) in Taiwan. Through the project cooperation, we are endeavoring to implement the proposed method for the assessment of the phase adjustment performance through open wye-open delta transformers. The outcomes gained from this study validate the feasibility of the proposed approach for balancing the phase operation of a power system, making it effective for electric power operation improvement applications. Reliability of scenario assessment becomes the major concern at this time. Further validation of this practical system will be reported in the near future.

Acknowledgments: The authors are greatly indebted to the Institute of Nuclear Energy Research for their valuable operating experience. Financial support of this paper under the contract number of MOST 104-3111-Y-042A-055 of Ministry of Science and Technology (MOST), Taiwan is appreciated.

Author Contributions: Ting-Chia Ou made substantial contributions to conception and plan; give final approval of the version to be submitted and any revised version. Wei-Fu Su handled the project. Xian-Zong Liu performed the experiments and conducted simulations; analysis and interpretation of data. Shyh-Jier Huang supervised the research throughout. Te-Yu Tai assisted analysis of algorithm.

Conflicts of Interest: The authors declare no conflict of interest.

\section{References}

1. Chang, C.Y.; Yu, J.S.; Chen, C.S. Effects of Open-Wye/Open-Delta Transformers on the Operation of Distribution Systems. Electr. Power Syst. Res. 1986, 10, 167-174. [CrossRef]

2. De Armas Teyra, M.A.; Gonzalez, G.G. Selection of Asymmetrical transformers banks with emphasis in losses and efficiency. IEEE Trans. Lat. Am. 2010, 8, 678-684. [CrossRef]

3. Santoso, S.; Dugan, R.C. Experiences with the New Open-Wye/Open-Delta Transformer Test Cases for Distribution System Analysis. In Proceedings of the IEEE Power Engineering Society General Meeting, San Francisco, CA, USA, 16 June 2005; Volume 1, pp. 884-889.

4. Tsay, M.T.; Chan, S.Y. Improvement in system unbalance and loss reduction of distribution feeders using transformer phase rearrangement and load diversity. Int. J. Electr. Power Energy Syst. 2003, 25, 395-401. [CrossRef]

5. Chen, S.L.; Li, R.J.; Hsi, P.H. Traction system unbalance problem-analysis methodologies. IEEE Trans. Power Deliv. 2004, 19, 1877-1883. [CrossRef]

6. Chua, K.H.; Lim, Y.S.; Taylor, P.; Morris, S.; Wong, J. Energy storage system for mitigating voltage imbalance on low-voltage networks with photovoltaic systems. IEEE Trans. Power Deliv. 2012, 27, 1783-1790. [CrossRef]

7. Li, K.; Liu, J.; Wang, Z.; Wei, B. Strategies and operating point optimization of statcom control for voltage imbalance mitigation in three-phase three-wire systems. IEEE Trans. Power Deliv. 2007, 22, 413-422. [CrossRef]

8. Lin, W.M.; Lu, K.H.; Ou, T.C. Design of a novel intelligent damping controller for unified power flow controller in power system connected offshore power applications. IET Gener. Transm. Distrib. 2015, 9, 1708-1717. [CrossRef]

9. Shahnia, F.; Majumder, R.; Ghosh, A.; Ledwich, G.; Zare, F. Voltage imbalance analysis in residential low voltage distribution networks with rooftop PVs. Electr. Power Syst. Res. 2011, 81, 1805-1814.

10. Ghahremani, E.; Kamwa, I. Optimal placement of multiple-type FACTS devices to maximize power system loadability using a generic graphical user interface. IEEE Trans. Power Syst. 2013, 28, 994-1000.

11. Ghahremani, E.; Kamwa, I. Analysing the effects of different types of FACTS devices on the steady-state performance of the Hydro-Québec network. IET Gener. Transm. Distrib. 2014, 8, 233-249. [CrossRef]

12. Lin, W.M.; Ou, T.C. Unbalanced distribution network fault analysis with hybrid compensation. IET Gener. Transm. Distrib. 2011, 5, 92-100. [CrossRef]

13. Ou, T.C. A novel unsymmetrical faults analysis for microgrid distribution systems. Int. J. Electr. Power Energy Syst. 2012, 43, 1017-1024.

14. Ou, T.C. Ground fault current analysis with a direct building algorithm for microgrid distribution. Int. J. Electr. Power Energy Syst. 2013, 53, 867-875.

15. Zhu, J.; Bilbro, G.; Chow, M.Y. Phase balancing using simulated annealing. IEEE Trans. Power Syst. 1999, 14, 1508-1513. 
16. Zhu, J.; Chow, M.Y.; Zhang, F. Phase balancing using mixed-integer programming. IEEE Trans. Power Syst. 1998, 13, 1487-1492.

17. Chen, T.H.; Cherng, J.T. Optimal phase arrangement of distribution transformers connected a primary feeder for system unbalance improvement and loss reduction using a genetic algorithm. IEEE Trans. Power Syst. 2000, 15, 994-1000. [CrossRef]

18. Huang, M.Y.; Chen, C.S.; Lin, C.H.; Kang, M.S.; Chuang, H.J.; Huang, C.W. Three-phase balancing of distribution feeders using immune algorithm. IET Gener. Transm. Distrib. 2008, 714, 55-65. [CrossRef]

19. Ding, F.; Loparo, K.A. Feeder reconfiguration for unbalanced distribution systems with distributed generation: a hierarchical decentralized approach. IEEE Trans. Power Syst. 2016, 31, 1633-1642. [CrossRef]

20. Askarzadeh, A. Bird Mating optimizer: An optimization algorithm inspired by bird mating strategies. Commun. Nonlinear Sci. Numer. Simul. 2014, 19, 1213-1228. [CrossRef]

21. Kersting, W.H. Distribution System Modeling and Analysis, 2nd ed.; CRC Press: Boca Raton, FL, USA, 2006.

22. NEMA National Electrical Manufacturers Association. NEMA Standard MG1-1987: Motors and Generators; NEMA National Electrical Manufacturers Association: Arlington, VA, USA, 1987.

23. TPC Taiwan Power Company. Technical Manual of Overhead Line Design for Distribution Systems; TPC Taiwan Power Company: Taipei, Taiwan, 2009.

24. TPC Taiwan Power Company. Interconnection Guideline of Renewable Energy; TPC Taiwan Power Company: Taipei, Taiwan, 2009.

25. Alajmi, B.N.; Ahmed, K.H.; Finney, S.J.; Williams, B.W. Fuzzy-logic-control approach of a modified hill-climbing method for maximum power point in microgrid standalone photovoltaic system. IEEE Trans. Power Electr. 2011, 26, 1022-1030. [CrossRef]

26. Haupt, R.L.; Haupt, S.E. Practical Genetic Algorithms, 2nd ed.; Wiley: Hoboken, NJ, USA, 2004.

27. Clerc, M. Particle Swarm Optimization; Wiley: Hoboken, NJ, USA, 2010.

28. Ou, T.C.; Chuang, S.J.; Hong, C.M.; Wu, R.C.; Tsao, T.P.; Chen, C.Y. Self-regulation ground faults model for microgrid distribution. ICIC Express Lett. Part B Appl. 2015, 6, 3225-3230.

29. Ou, T.C.; Hong, C.M. Dynamic operation and control of microgrid hybrid power systems. Energy 2014, 66, 314-323. [CrossRef]

30. Hong, C.M.; Ou, T.C.; Lu, K.H. Development of intelligent MPPT (maximum power point tracking) control for a grid-connected hybrid power generation system. Energy 2013, 50, 270-279. [CrossRef]

(C) 2016 by the authors; licensee MDPI, Basel, Switzerland. This article is an open access article distributed under the terms and conditions of the Creative Commons Attribution (CC-BY) license (http://creativecommons.org/licenses/by/4.0/). 